\title{
Specification and Design of Interactive Multimedia Services for Broadband Intelligent Networks
}

G. N. Prezerakos, I. S. Venieris

National Technical University of Athens

Department of Electrical and Computer Engineering

9 Heroon Polytechniou str, 15773 Athens, Greece

tel. $+3017722551 /$ fax +3017722534

e_mail: ivenieri@cc.ece.ntua.gr

\begin{abstract}
We describe a complete methodology for the creation of interactive multimedia services (IMM) over Broadband Intelligent Networks (IN), that covers all phases from verbal description of a service up to the point when the service is ready for deployment. The paper discusses alternatives for the distribution of the service logic in the IN/B-ISDN network elements as well as the specification of the messages flows across interfaces. SDL is used for the production of a formal description of the service which is mapped into a Service Logic Program and simulated with a Service Creation and Simulation Environment. Throughout the paper two services are used as running examples to demonstrate the proposed methodology: a Video on Demand ( VoD) service which is a novel IN service and a Broadband Video Conference Service (B-VC) which is based on an existing implementation originally targeted for a TCP/IP network platform.
\end{abstract}

Keywords

Broadband Intelligent Network, Multimedia Service design. 


\section{INTRODUCTION}

In the last few years the area of Intelligent Networks (IN) has been rapidly expanding on a world-wide scale. The resulting need for specification of the target IN architecture has led to the creation of a framework for the description and design of IN services referred to as the IN Conceptual Model (INCM). The INCM sets the guidelines for a set of evolving specifications called capability sets (CS) which define requirements in a variety of IN-related aspects such as service creation, service management, service interaction, network management, service processing and network interworking.

The basic principles of IN service specification have been laid out by ITU-T in (ITU-T I.210) for the narrowband environment. Moreover the IN Conceptual Model is described in detail in (ITU-T Q.1201) as consisting of four planes : the service plane, the global functional plane, the distributed functional plane and the physical plane. The Service Plane is primarily used for representing services from the user's point of view. In that context the various services and service features are described independently of the actual implementation. The Global Functional Plane (GFP) is primarily used for representing services from the service designer's point of view. Service functionality is assumed to be included in units called Service Independent Building Blocks (SIBs) which are independent of how the functionality is distributed across the network. Thus the network is seen, from that plane, as a single entity. SIBs can be chained together in various combinations in order to realise services and features specified in the Service Plane. The way these SIBs are combined in the process of service creation is called Global Service Logic (GSL). The implementation of the GSL is called a Service Logic Program (SLP). The Distributed Functional Plane (DFP) is primarily used for representing services from the network designer's and service provider's point of view. Service functionality is divided between various network units referred to as "functional entities" while these entities are connected by means of information flows referred to as "relationships". Functional entities are independent of how the functionality is physically implemented in the network. From this plane the network is viewed as being comprised of functional entities connected by information flows. The Physical Plane is primarily used for representing services from the network operator's and equipment provider's point of view. The physical architecture of an IN structured network is described in terms of physical components, referred to as "physical entities", and the interfaces between them. One or more functional entities and relationships from the DFP can be mapped to a physical entity or interface respectively in the physical plane.

The definition of such a solid theoretical background meant to address the core problem concerning the introduction of the IN concept that is flexibility in design and deployment of telecom services as well as customisation of the service. The 
combination of these advantages can lead to reduced development time and cost thus permitting the service providers to keep in pace with market needs. Nevertheless the process of service design and deployment is still in many cases extremely time consuming a fact that also leads to the consumption of significant financial resources. The introduction of interactive multimedia services which in turn demand increased bandwidth and more complex features belonging to the currently non-standardised CS-2 / CS-3 only makes the situation more complicated.

Various interesting aspects of the IN service design process can be found in the literature (Thörner, 1994), (Nyeng, 1993), (Olsen, 1995). Existing research in the field of IN service development proposes the adoption of the Specification and Description Language (SDL) as a key tool during such a process. Several approaches can be distinguished:

a) The use of SDL as a stand-alone description, simulation and verification tool for IN services. Approaches of this kind range from simple ones (Nyeng, 1993), to more sophisticated (Doza, 1990) to quite complicated where several models of IN services are examined (Kelly, 1995).

b) The use of SDL for the production of IN service source code targeted to a software development platform such as CORBA (Olsen, 1995).

c) The use of a Service Creation and Simulation Environment (SCSE) which may also incorporate the specification phase by including an SDL based specification tool (Niitsu, 1992), (Bosco, 1994). An SCSE has the ability of producing the actual Service Logic code that runs on the IN network therefore it is considered by many developers as a crucial element in the service development process.

The use of SDL as a stand-alone tool adopts the object oriented concepts that have become available with SDL-92. The object oriented methodology offers an elegant way of describing a service, incorporating a new service feature into an existing service and incorporating a new service in an existing description. Moreover by the use of SDL compatible validation and simulation tools the resulting system can be checked against errors in design and operation. On the other side describing a service in such a manner makes the transition from the prose to the formal description non-straightforward. This situation arises from the fact that the prose description is based on a telecom based view of the service while the object oriented approach is software engineering oriented. This is also the reason while such a design demands excessive effort from the developer's side in order to be understood. Even in the cases where such a service has been validated and simulated without problems this method does not present the developer with an smooth path towards the actual implementation since a direct mapping from the SDL description towards an SCSE is not provided.

The use of SDL for generating $\mathrm{C} / \mathrm{C}++$ code targeted to a platform such as CORBA is a more viable alternative although it presents some drawbacks like : SDL specification of the system following very strict rules, adaptation of the resulting $\mathrm{C}$ code to CORBA, involvement with implementation details that would 
be transparent if an SCSE was used. As a general remark it could be stated that systems designed in that manner require a tedious debugging process before the production of a high-level language code is attempted while the produced code always requires extensive changes in order to be downloaded to the IN network.

ITU-T has already standardised some IN service design elements (i.e. SIBs) which could be used for the specification of an IN service and for its design provided that a relevant design tool exists. Unfortunately SIB based design is not supported by any major commercially available development tool therefore the potential developer still faces the problem of providing a mapping from the SIB based specification to the selected development platform. Moreover it will be demonstrated in this paper that the specification of a telecom service includes aspects from both the Global and the Distributed Functional Planes, a fact which cannot be dealt with by using SIBs which are targeted to the GFP only. On the other hand SDL by offering the capability of mixing features from both functional planes is a more promising candidate for the specification phase of a service.

The approach discussed in this paper attempts to combine SDL service specification with a commercially available IN SCSE. The use of such a software platform renders the remaining INCM planes transparent to the potential service designer who has to bear in mind only the requirements of the GFP. Any enhancement regarding specific service functions or parts of the service logic can be easily accommodated by imposing high-level modifications to the existing design. Another advantage is that the stage of producing $\mathrm{C} / \mathrm{C}++$ code and then modifying it to suit the specific system architecture does no longer exist since the platform based service design is directly incorporated into the IN network. The main difference from the (c) approach presented earlier is that SDL specification is treated independently from the SCSE which is used for development. The reason is that a lot of commercial development environments lack a specification verification module (SDL compatible or not) and the ones that do present the potential developer with an additional level of usage complexity. As it will be shown in this paper treating the specification outside the creation environment does not pose any significant problems during the development phase of the service.

After a detailed presentation of the proposed methodology we proceed with two examples of service creation. The first is a typical IMM service of Video on Demand (VoD) service which serves as a case of an originally non-existent interactive multimedia service which is $100 \%$ compliant with the IN environment. The other is the Broadband Video Conferencing Service (B-VC), an existing service with special call and data handling requirements originally targeted for a different network platform which is migrated to the IN environment. The latter example demonstrates the advantages of the IN concept in aspects regarding cost minimisation of incorporating applications developed having in mind varying telecommunication concepts into the IN concept.

This paper is organised as follows: Section 2 presents an overview of the proposed methodology. Section 3 covers the stages of prose description and 
functional architecture as well as the interface specification in the Distributed Functional Plane. Section 4 discusses the issue of formal service description in SDL. Section 5 covers service design by means of commercial development platforms. Finally, conclusions are presented in Section 6.

\section{METHODOLOGY FOR SERVICE DESIGN AND IMPLEMENTATION}

It is a well known that telecommunication applications are of a distributed nature. Distribution can be seen in terms of players as well as in terms of systems. From one point of view the service logic is distributed across the various network elements which are actively involved in the life cycle of a service. From a different point of view service capabilities depend upon the various players in the field of design and deployment i.e. service users, service developers, service providers, telecom operators and equipment providers. Thus the problems one faces with the implementation of a telecom service, having in mind the aforementioned objectives, are mainly concerned with the impact of each player on the service, the distribution of service logic across the various systems, the interworking between these systems, the definition of a common description which is both accurate as well as understandable and the evolution of such a service description into a Service Logic Program. Provided that the network infrastructure is quite invariable, thus rendering the telecom operators indifferent, the impact of the rest of the players has to be examined.

The service user group is of course a key player in the sense that it is the ultimate judge of the success or failure of a new service or of a new feature added to an existing service. The user's view of a service should always be considered as the starting point of every service creation effort. Even in the cases where a market demand does not yet exists but its creation is attempted by a service provider, a description of how a potential user might envisage a service should always be provided.

The service developer and the service provider are, if not identical, at least closely cooperating organisations. The service provider is responsible for merging the user's demands with its own preferences and the target $\mathrm{IN}$ architecture in order to produce a formal service description. The service developer maps this description to a set of service building blocks that are used to describe the service logic. The current trend is the usage of a Service Creation and Simulation Environment which facilitates the development process by the aid of visual programming tools and object-oriented techniques.

Finally service logic has to be tested, debugged and loaded to the various network nodes. In theory if the development process is conformant to the international standards only minor modifications are required in order for the service to become operational. In practice, since broadband capability sets are currently under standardisation, the existing equipment capabilities (switches, workstations etc.) 
have to be taken into account while the development phase is still ongoing. Due to its complex nature this area is left for further research and is out of the scope of the paper.

The proposed methodology is presented in Figure 1 in the form of discrete stages:

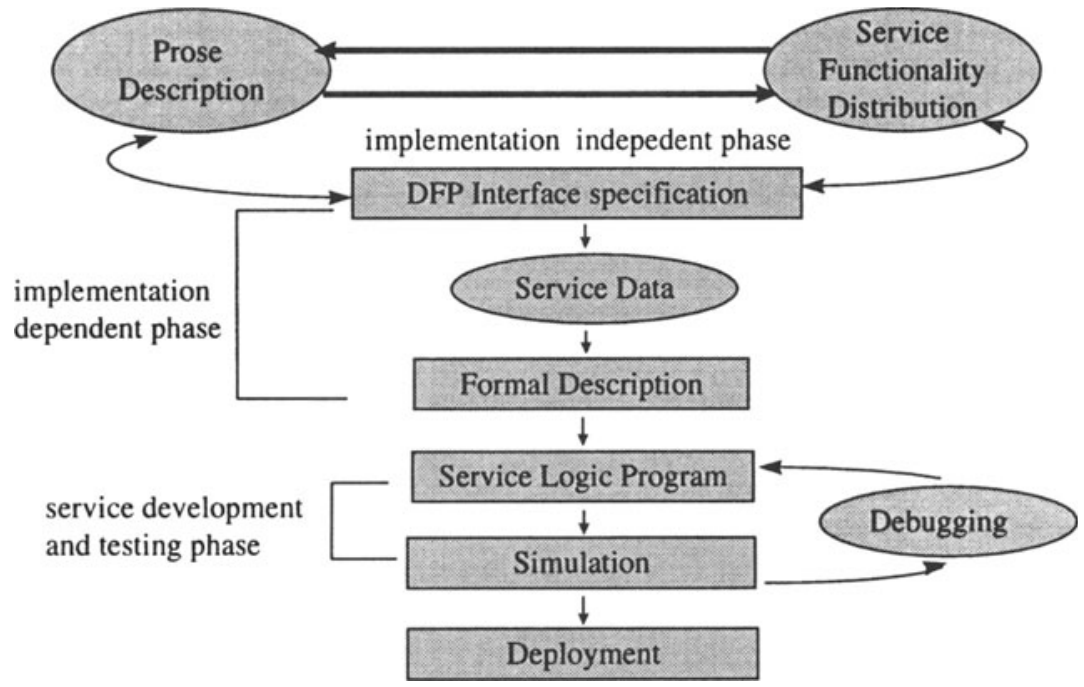

Figure 1 Specification and Design Methodology.

A prose description of the service from the user's point of view is the first stage of service creation. Based on the prose description a definition of how the service functionality will be distributed is carried out. This is an attempt to define which networks elements will be involved in the service and up to what degree and also the parts of service logic that they will host. This stage is followed by the interface specification in the DFP. By aggregating the previously acquired information along with a definition of the data structures required by the service a formal description of the service is produced in SDL. This description is consequently transformed into Service Logic Code and simulated with a SCSE. Finally the service, after being successfully tested and tuned, becomes operational.

The impact of each stage upon IN service creation is also examined from a practical standpoint by using the two real-life IN services already mentioned: the Video on Demand (VoD) and the Broadband Video Conference (B-VC) service. These services have been selected bearing in mind that they are broadband interactive multimedia services, comprehensible within the telecom community with a development process that presents difficulties similar to many telecom services and also that their service logic contains modules that can be reused in other IN based services.

The $\mathrm{VoD}$ is a service that enables the user to connect to a video server and view a movie or a television program (or in general any audiovisual information) from 
home by using a TV set equipped with a set-top box. The assumption will be made that no realisation of this service previously existed therefore the service is directly targeted to the IN architecture. The version of the VoD service examined in this paper is based on (Vezzoli, 1996). The B-VC service facilitates the communication between groups of users by enabling them to setup conferences in which each of the conferees can participate from his own premises. Originally targeted to a TCP/IP network the service is migrated to the IN architecture by trying to retain as many of its existing features as possible while adding IN functionality. The version of the BVC service examined in this paper is based on (Brandt, 1997). The service logic for both services is presented in detail with emphasis given in the GFP and the DFP. The flow of the service is depicted by means of Broadband Intelligent Network Application Part (B-INAP) messages exchanged between the various network entities from the point of service request until the point where the service logic is terminated.

\section{SERVICE DESCRIPTION AND FUNCTIONAL ARCHITECTURE}

\subsection{Prose description}

The prose description of a service from the potential user's point of view can be dealt with various software engineering strategies. The following are considered as key steps in such an approach:

It is better to decompose the life of a service session into consequent discrete stages. Apart from facilitating the description itself, such a decomposition makes the transition to subsequent phases of the development process easier.

A service scenario should be given for each of these service stages. The scenarios are structured on the basis that the service logic prompts the user for input, the user responds and the service reacts accordingly by following one of the possible service logic execution paths. Implementation specific details as well as the functional architecture are not considered at all during this phase. The only fact that is taken into account is that the user possesses a multimedia terminal of some kind connected to an underlying broadband network infrastructure.

In a summarised description of the $\mathrm{VoD}$ service in terms of stages, the following stages can be identified: Service Provider Selection, Authentication-Authorisation, Type of Content Selection, Address Translation, Content Selection. In a summarised description of the B-VC service in terms of stages, the following stages can be identified: Conference creation, Static Conference Management, Add user to conference, Conference Establishment, Join conferee, Leave conference, Close conference and Dynamic conference management.

According to (ITU-T, Q.1213) each Service Logic Program is composed by a series of interconnected SIBs. Service execution always commences from the Basic Call Processing (BCP) SIB and proceeds to other SIBs through Points of Initiation (POIs). The service is terminated in a similar fashion when the execution returns to 
the BCP SIB through Points of Return (PORs). Following along this line, services can initially be represented by diagrams showing the interconnection between the various service stages and the Basic Call State Model (BCSM) through POIs and PORs.

The relevant diagram for the $\mathrm{VoD}$ service is given in Figure 2 and for B-VC service in Figure 3:

Video on Demand

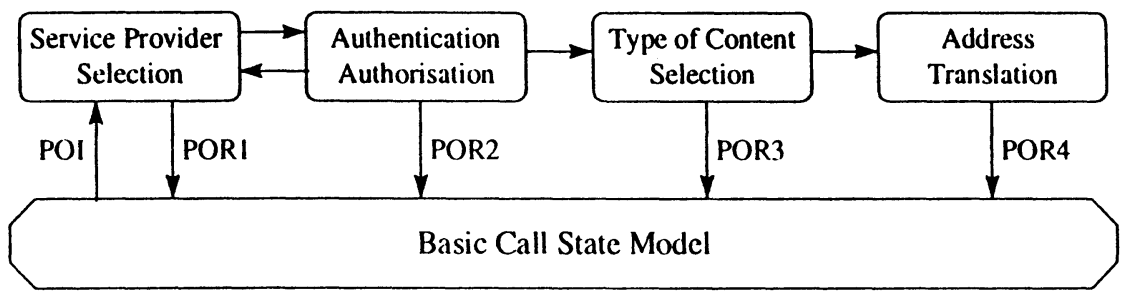

Figure 2 Decomposition of the VoD Service.

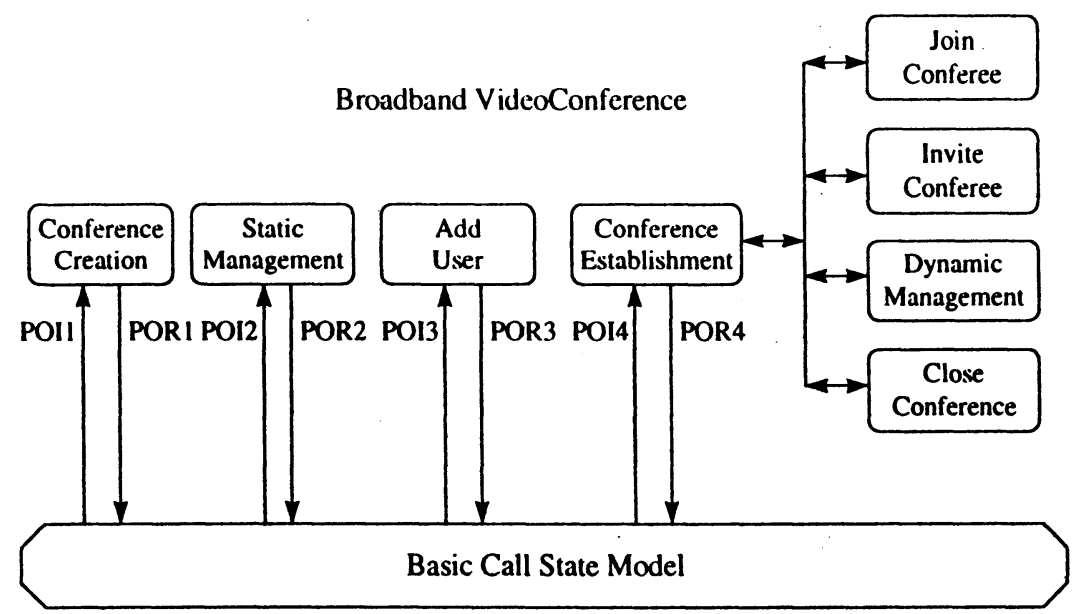

Figure 3 Decomposition of the B-VC service.

These diagrams, since they comprise aspects of both the service plane and the GFP, can serve as the link between the prose description phase of the service and the SDL specification.

\subsection{Service functionality distribution}

The IN architecture is based on information exchange between the following network elements: the Broadband Service Control Point (B-SCP), the Broadband Service Switching Point (B-SSP), the Service Data Point (SDP), the Broadband Intelligent Peripheral (B-IP) and the Customer Premises Equipment (CPE). In the context of the standardised approach towards IN service development the Service 
Logic resides completely inside the B-SCP, the B-SSP realises the service's switching functionality, the SDP is in charge of database transactions, the B-IP provides the resources needed (audiovisual announcements) for communication with the CPE. The CPE functionality is usually restricted to the realisation of a User Network Interface (UNI) signaling stack although the existence of intelligent CPEs is not excluded.

The concept of realising the Service Logic completely inside the B-SCP may be proven vulnerable when one proceeds to real life implementations. In practice the B-SCP is a workstation which depending on the load imposed by service execution may not be able to handle the required number of transactions. A viable alternative, if the involvement of a more powerful machine severely affects the cost curve of the service, is to split the service functionality between the B-SCP and the B-IP by moving significant parts of the service logic there.

Moreover the need for adding service logic functionality to the B-IP arises from the fact that the standardised approach that was mentioned earlier is a heritage from the days of narrow-band services where an audio message was played to the user and the required user response was entered by pressing buttons in the telephone keypad. On the other hand broadband interactive multimedia services are expected to compose several different media in order to interact with the user and acquire a user response of similar nature thus rendering the duties of the B-IP far more complex than the transmission of audio messages. Communication between the user and the IN service can be handled by integrated parts of service logic that reside in the B-IP (nevertheless still activated by the B-SCP). Aware of this situation ITU-T has attempted to standardise such an approach in (ITU-T, Q1228). For the needs of this paper, following ITU terminology, we will be referring to "scripts" and to "scripts functionality". Selected parts of the VoD service will be presented by using the "scripts" approach.

User requests would be best transferred to the B-SCP by the User To Service Interaction (UTSI) feature. Because this feature is not available in the services demonstrated in this paper, an alternate approach which uses the B-IP as a relay for signalling between the CPE and the service logic was chosen. When user input is needed the logic issues a PromptAndCollectUserInfo request. All user requests are send to the B-IP over a connection between CPE and B-IP. The request is then send to the B-SCP as the CollectedUserlinfo message.

The topology of the VOD service, presented in Figure 4, is aligned with the above line of thought. 


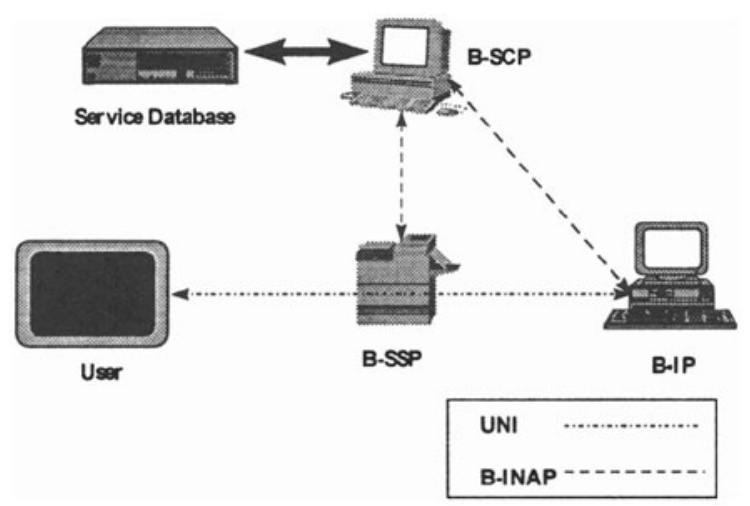

Figure 4 VoD Topology.

The B-SCP/B-IP and B-SCP/B-SSP communication is achieved through the BINAP layer of the IN protocol stack. The Service Database is contained within the B-SCP. The User/B-IP communication is achieved through UNI signalling and the B-IP handles the User/B-SCP communication by appropriately routing the respective messages.

In cases where an existing service is adapted to the IN architecture (as the B-VC service) the B-IP can undertake an additional role towards the facilitation of the migration process. Since the B-IP is used as a router that conveys the necessary information between the User and the B-SCP, the protocol stack that handles the user to service communication can be retained in the new environment. Moreover several service functions can become B-SCP independent and completely realised within the B-IP. It is easier to port major parts of the original application to the BIP (which in most cases is a workstation compatible with the original implementation) than to the B-SCP which may operate under a different software / hardware platform.

The B-VC service presents the additional complexity of how it handles conference data and user connections. During certain operations, such as conferee addition, certain database records should remain locked to prevent other users performing the same or different functions from altering them simultaneously. The most effective way to handle such a situation is to use database locking in the record level. Assuming that special facilities such as record locking are not always available within the B-SCP then the only alternative is to duplicate the Service Database Tables (SDTs) in the B-IP. Database synchronisation can be achieved by moving records between the B-SCP and the B-IP only when it is dictated by a B$\mathrm{VC}$ operation. The resulting service topology is presented in Figure 5. 


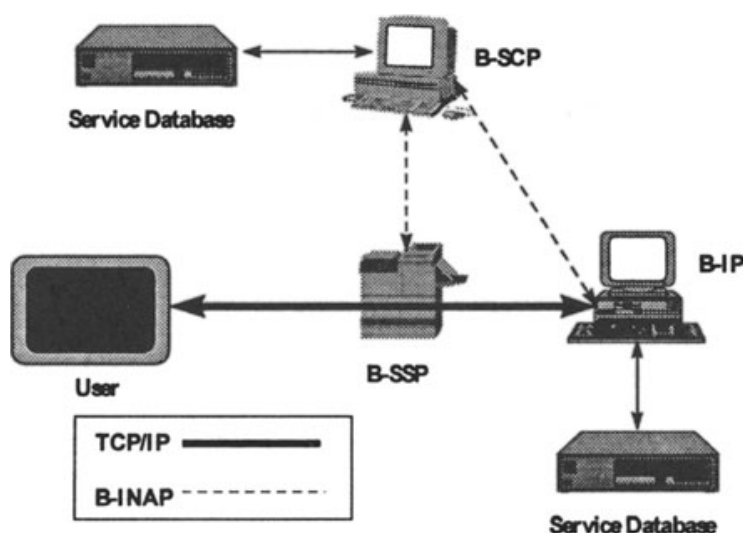

Figure 5 B-VC Topology.

The Service Database is contained in both the B-SCP and the B-IP. The User/BIP communication is achieved through the initially used TCP/IP protocol and the BIP handles the User/B-SCP communication by appropriately routing the respective messages.

The need for interaction between Service Logic Program instances arises in operations like 'Join Conferee' where the conferee that wishes to join a running conference activates a Service Logic Instance which consequently should communicate with the Service Logic Instance of the running conference and negotiate the conferee's participation. When the conferee is joined the user initiated Service Logic Instance must be dropped. It is the duty of the B-IP to undertake the correlation of the two instances.

\subsection{Interface specification in the Distributed Functional Plane}

When the service logic allocation to the various network elements has been completed the interfaces between them must be specified prior to a formal service description. This includes the information flows connecting the various elements consisting of messages, data types and data structures that will be utilised by the BINAP layer of the IN protocol stack.

Summarising the presentation in section 3.2 , the actively involved network elements in any approach are the B-SCP and the B-SSP. The SDP is usually a database engine incorporated in the B-SCP and it is not considered as a separate entity. The service's database design though is an important aspect of the overall development process and a research field of its own right. This paper address only the problem of locating the SDTs, according to the selected IN service functionality distribution. It is obvious that a minimum prerequisite towards a formal service specification is the definition of the SCP/SSP and SCP/IP interfaces along with the corresponding information flows. 
The scripts approach to the VoD service demands that the service logic is divided between the B-SCP and the B-IP. Initially the user is first connected to the B-IP, then the B-SCP demands a script execution from the B-IP. The script is executed and finally the result is conveyed to the B-SCP. An evolution of the Information flows for a VoD service, proposed in (Vezzoli, 1996), towards the scripts approach is presented in Figure 6 for the Service Provider Selection stage:
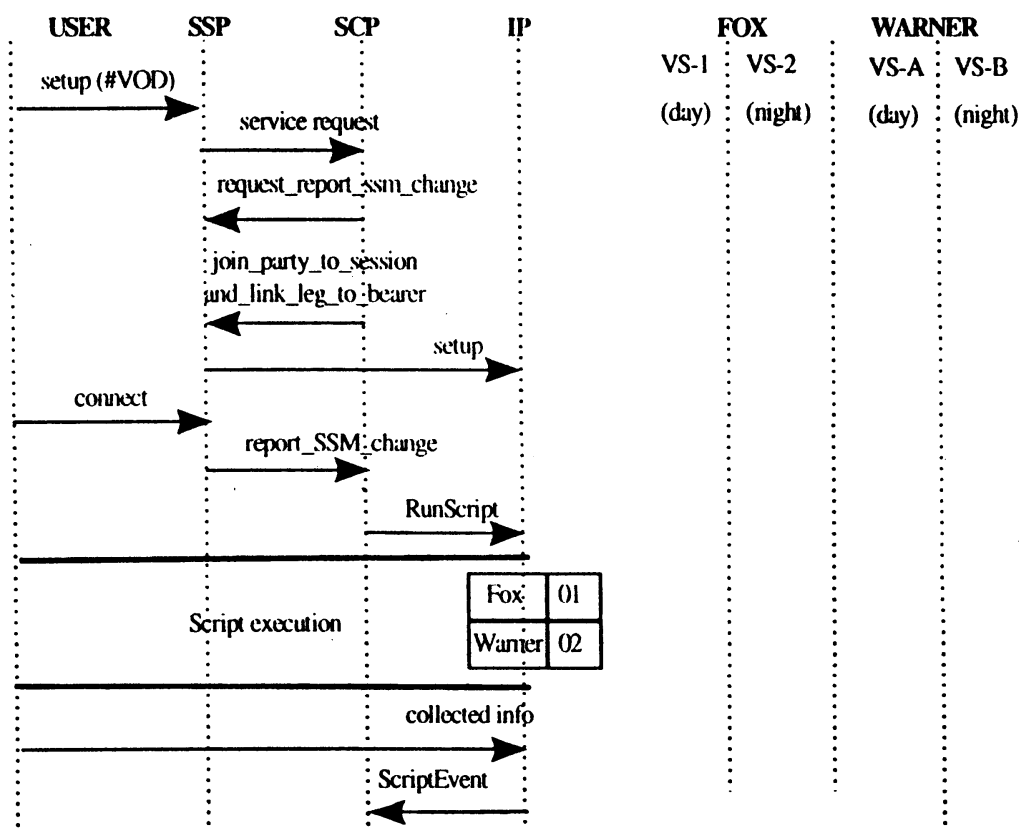

Figure 6 VoD Information flows.

Data structures can have a great influence on IN service design. A properly designed service should allow the potential user to exploit the offered functionality to the greatest extent while bearing in mind that different users usually require different treatment from each IN service. A useful starting point might therefore be to identify Subscriber and user profiles that would determine which parts of the service functionality are to be used. The data handed by the service logic could be subdivided as: (i) service data (e.g. fixed data and announcements), (ii) management and subscription data (e.g. Subscription profiles), (iii) user data (e.g. user profiles), (iv) call instance data (e.g. counts against unauthorised access attempts)

Service data and call instance data may be identified during the formal specification stage without inducing major drawbacks in the development process. On the other hand management, subscription and user data should have been defined in an earlier stage since an incomplete or erroneous definition of these data structures may result to significant modifications during the implementation phase 
thus causing severe delays service development and deployment. Therefore it is imperative that a detailed description of (ii) and (iii) exists prior to the service's formal specification.

The location of each SDT is determined by the location of the corresponding Service Logic Program function. As a general rule, aiming at the minimization of data transfers between the various network nodes, SDTs are located at the same network node where the respective Service Logic is executed.

\section{FORMAL SERVICE DESCRIPTION}

The stage of formal service description attempts to combine requirements defined within the previous stages and produce a solid service description that is understandable by both the service provider and the service developer and "freeze" the specification process. The use of SDL for this purpose is suggested because SDL is an international standard tailored for the description of telecommunication systems and in case a SCSE does not exist then additional tools can be used for verification and simulation purposes.

There are various approaches towards the representation of a telecommunication service via SDL mainly based on the possible uses of such a description. In our case the SDL specification meant to produce a detailed presentation the service logic from the telecom point of view instead of the software engineering point of view while combining of both the DFP and GFP service functionality. In the context of this paper SDL is not used as an implementation language therefore its code generation and simulation/validation facilities will not be used. Nevertheless the proposed system schematics remain aligned with the recommendations described in (ITU-T , Z.100).

Each IN service is represented by the SDL system structure. This way each IN service is described in an independent self-contained manner. The network elements involved in service logic execution are represented by the SDL block structure. These structures may be further decomposed to objects in the sub-block level if necessary. However decomposition into several layers may only be necessary for complex services or for groups of services heavily interacting with each other.

The connection between the various network elements is represented by SDL channels. Messages exchanged between IN network elements are represented by the SDL signal structure. Each service stage, described in the prose description, is represented by an SDL process. Service stages that may be activated within the scope another stage are represented by SDL procedures. Procedures are also used to group pieces of SDL code that are frequently repeated in various points of a service e.g. the ConnectUsertoIP procedure. At each block or sub-block, the interfaces and attributes of each object should are defined accompanied by textural comments relating to the function to be performed. 


\subsection{VoD service description - scripts approach}

VoD service functionality can be distributed between the B-SCP and the B-IP in a number of ways. The approach presented in the following figures assumes that Service Provider Selection, Authentication-Authorisation and Type of Content Selection stages are performed in the B-IP. These operations have been realised as scripts and are completely independent entities which handle all possible service logic outcome internally. Their only relation to the B-SCP is that they are activated by it and that they are obliged to notify script termination by returning an appropriate code. After successful completion of script execution Address Translation is performed in the B-SCP and the B-SCP instructs the B-SSP to connect the user to the VS. Upon successful connection to the VS the SLP terminates.

The proposed functionality distribution results in relocating the SDTs to the network element that handles the respective database operation. The Service Providers, User Profiles, Type of Content SDTs have now been moved to the B-IP. The Routing SDTs remain in the B-SCP. An overview of the system can be seen in Figure 7.

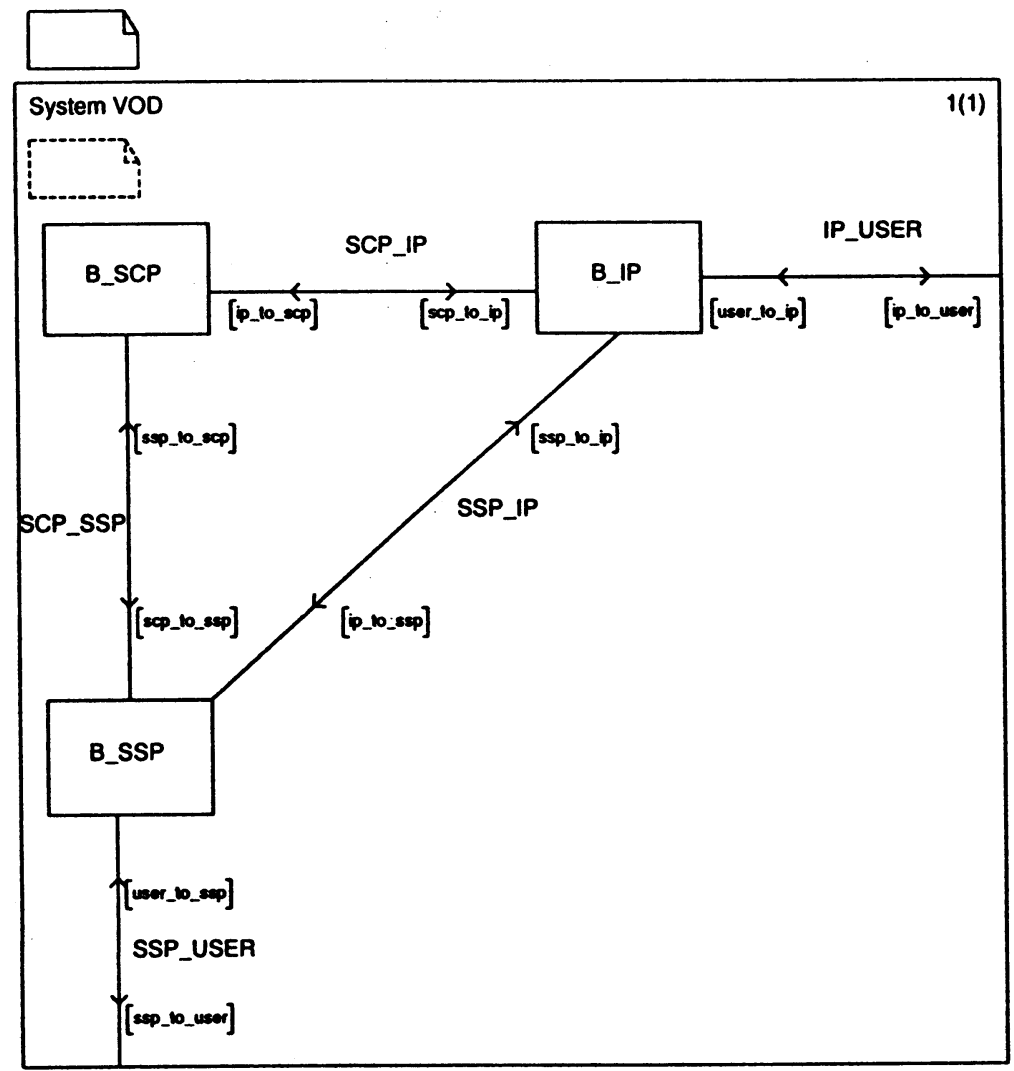

Figure 7 VoD service overview in SDL (scripts approach). 
The service logic in the B-SCP is included in the Service_Logic_Program process. The portion of this process regarding the Service Provider Selection is presented in Figure 8.

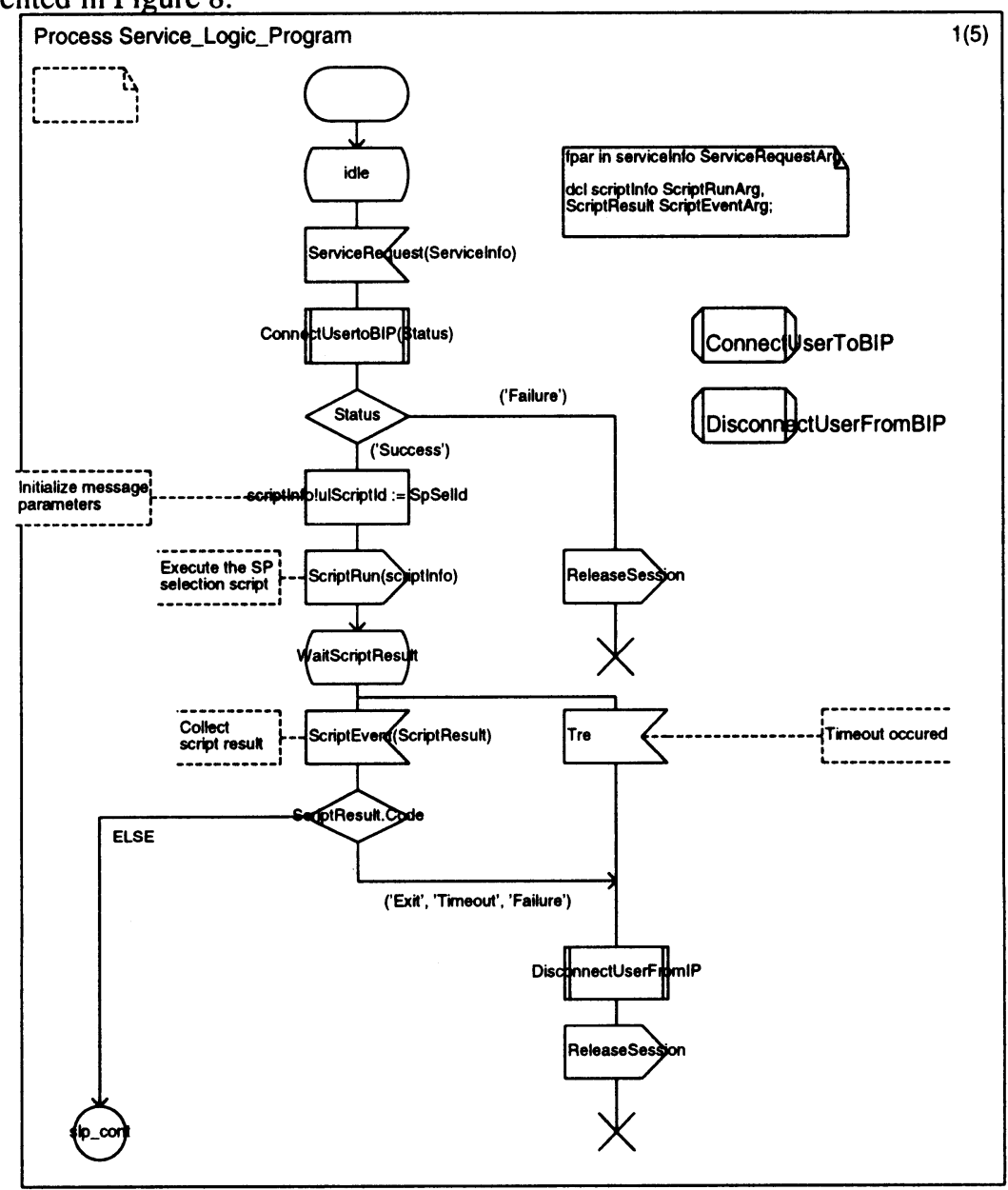

Figure 8 VoD Service Provider Selection in SDL (scripts approach).

The general structure of the corresponding B-IP service logic is depicted in Figure 9. The ScriptManager process awaits an activation message from the B-SCP. Upon receipt it executes the corresponding script. Notice that since scripts are called from inside a process they are realised as procedures. 


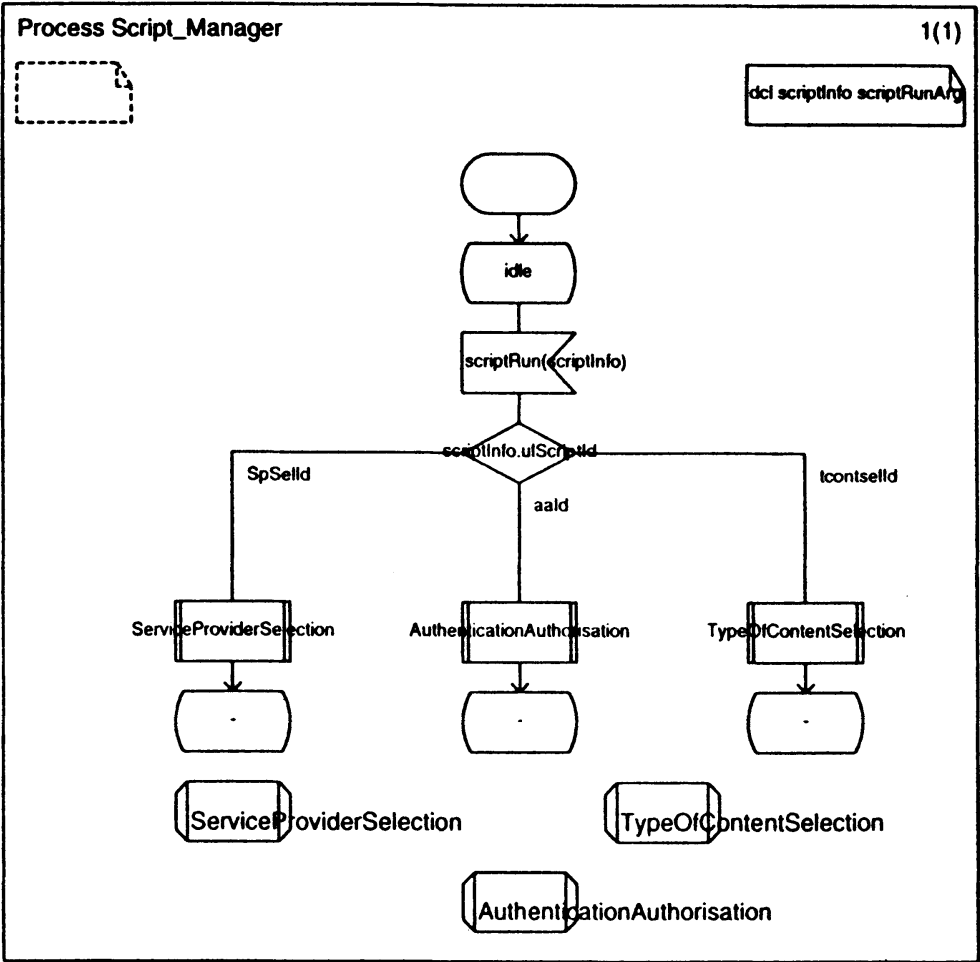

Figure 9 Description of the VoD B-IP Service Logic in SDL.

\subsection{B-VC service description}

The B-VC has been described as a "hybrid" service since it is not $100 \%$ IN compatible. The system overview diagram is identical to that of Figure 7 . The Conference Creation process has been selected as a typical example of B-VC service logic and is described in figures 10,11 and 12 .

The Conference Creation process is invoked when a user dials the appropriate IN number. A conference creation request is issued and the following procedure is activated : The User dials the IN number indicating that he wishes to be added to the conference. Initially the service logic checks whether the user is an authorised one or not. If the user is indeed authorised then the B-SCP requests from the B-SSP to connect the user to the B-IP.

The B-IP is the network element that will undertake the role of correlating the conferee connections during the lifetime of an active conference. If the connection is successful then a user - IP dialogue takes place by which the profile of the conference to be created is determined. At the same time the B-SCP waits a notification message from the B-IP.

If the notification message from the B-IP arrives in time then the service logic requests from the B-IP to lock the conferences database and create an entry for the requested conference. The B-IP attempts to execute the request then responds by 
returning a status code. If the conference record has been created successfully then the B-SCP updates the local copy of the database. The B-SCP then requests from the B-IP to unlock the conference database. The B-IP once more attempts to perform the requested action, returns a status code and the service instance is terminated.

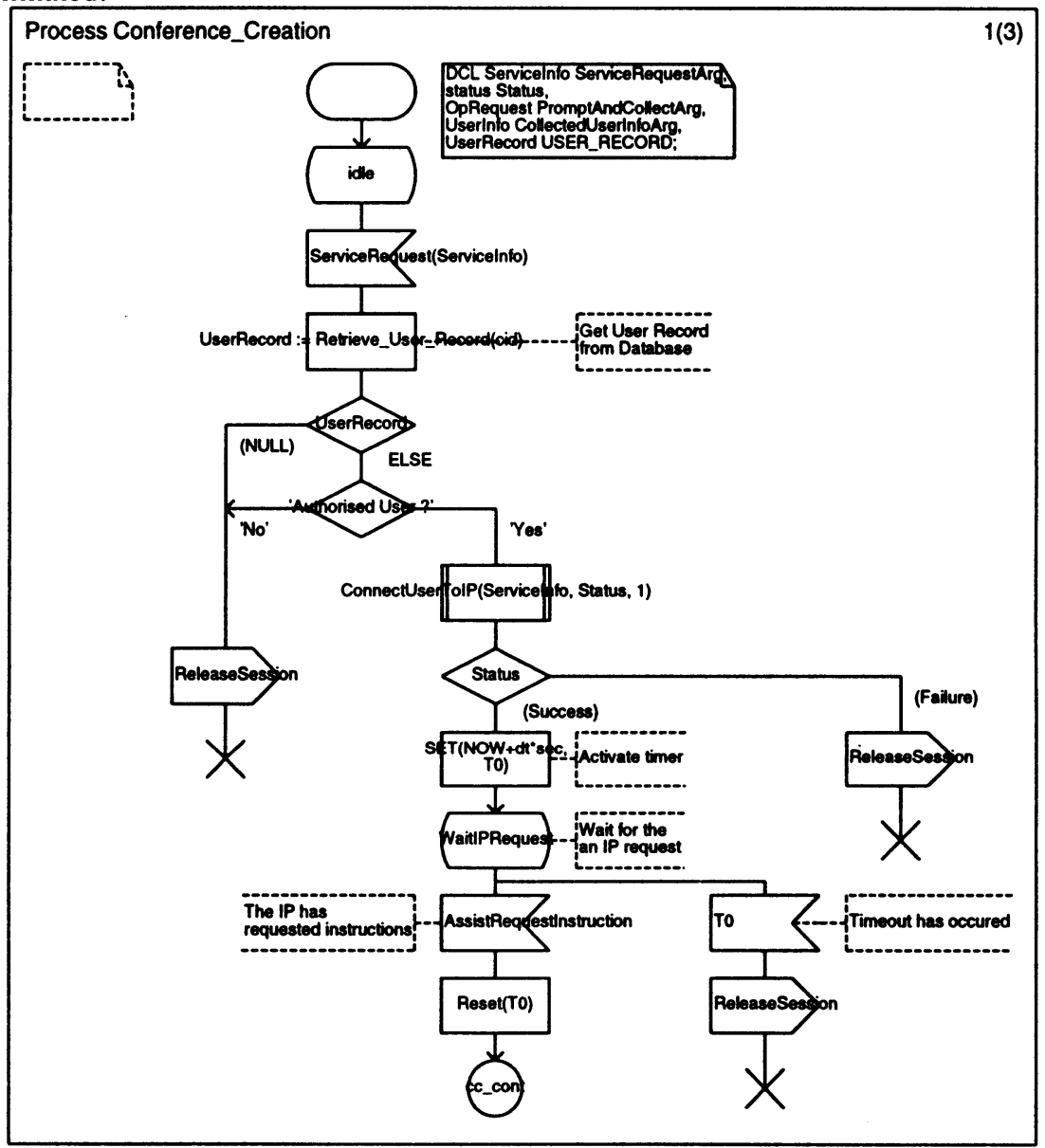

Figure 10 B-VC service Conference Creation SDL description (page 1).

The strategies used demonstrate the flexibility of the IN architecture and can be understood by consulting the SDL schematic. First of all, the application parts that handle user to service interaction remain almost intact when the service is ported to the new environment since the B-IP is used as a message router based on the existing UNI protocol. The call correlation needed for complex multi-user services (like the B-VC service) is taken care of by the B-IP and this approach results to slight modifications with respect to the initial version of the application. Also the use of the twin databases scheme compensates for the lack of record locking 
facilities in the B-SCP. In the majority of the existing scenarios only identifiers need to be transferred between the B-SCP and the B-IP.

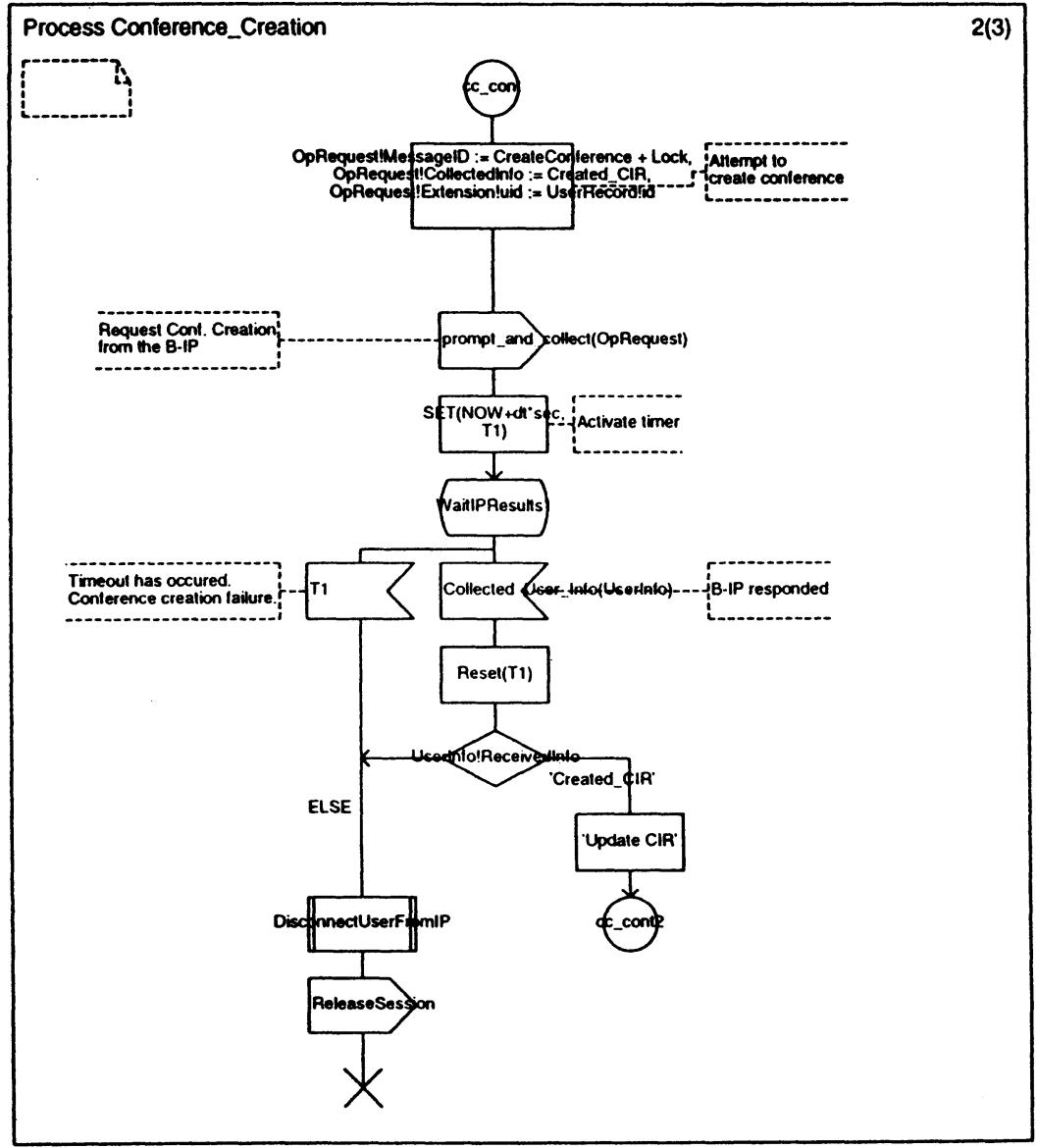

Figure 11 B-VC service Conference Creation SDL description (page 2).

Finally the PromptAndCollect as well as the CollectedUserlnfo B-INAP messages have been extended so as to accommodate the B-SCP/B-IP communication. More specifically, the MessageID and CollectedInfo field of the PromptAndCollect message have been extended in order to carry the requested B-IP operation. An extra field, namely Extension, has been added divided into the CIR, UIR, CID and UID fields. These fields contain, whenever is necessary, the Conference Information Record, User Information Record, Conference Identifier and User Identifier respectively. Apart from user input the Receivedinfo sub-field of the UserInfo field of the CollectedUserInfo message is also carrying B-IP responses and success/failure codes. 


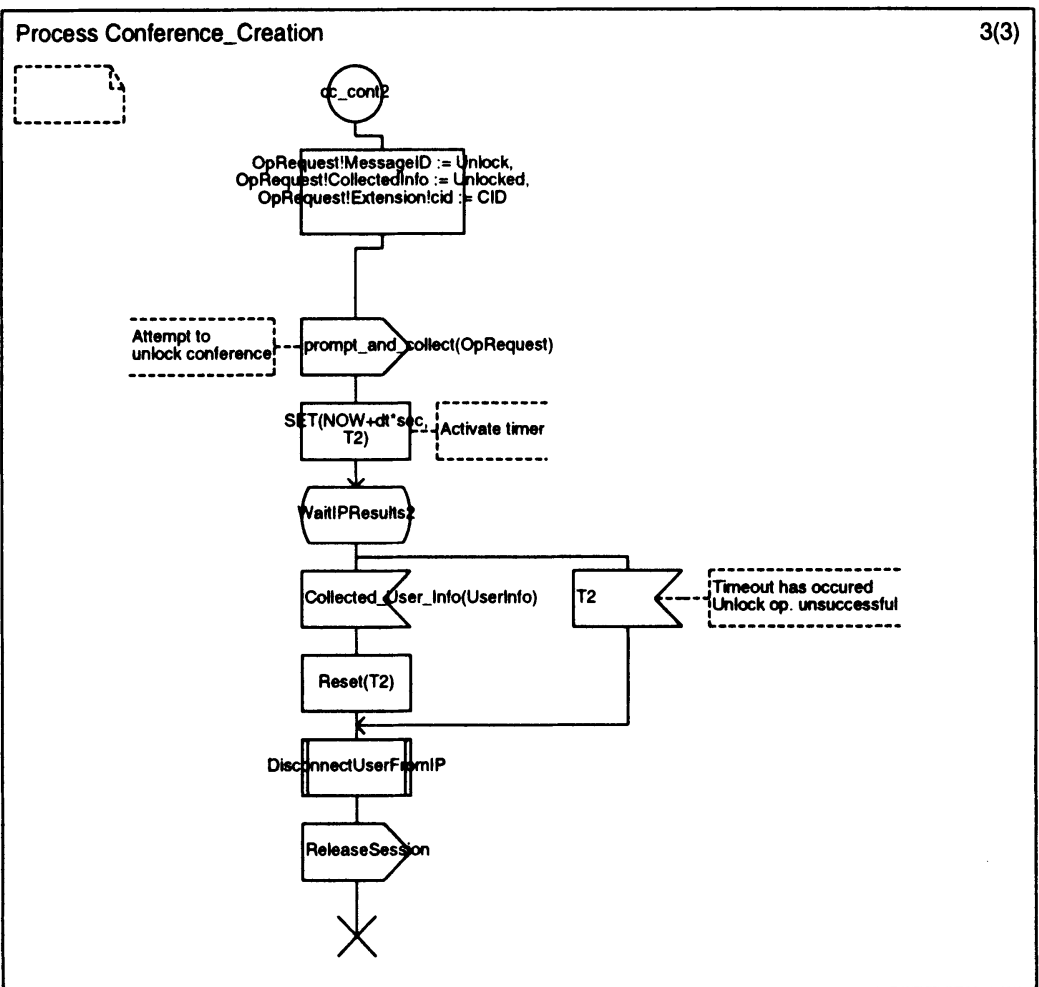

Figure 12 B-VC service Conference Creation SDL description (page 3).

Such an approach, no matter how flexible, presents certain disadvantages: The number of messages exchanged between the B-SCP and the B-IP is high, transfer of database records between the various network nodes is not completely avoided and the SLP is obliged to perform extensive check of the B-IP return codes. Nevertheless, compared to the cost of rewriting extensive parts of the existing code in order for the application to be ported to a different telecommunication architecture, these compromises seem justified.

\section{SERVICE DESIGN}

This section deals with the actual development of the SLP as well as the simulation of the service which are the next design stages after the solidification of the formal specification. There are a few SCSEs available in the market. This paper presents a generic broadband SCSE which is loosely based on (GPT, 1996). Although the only standardised building element for an IN service is the SIB, the majority of SCSE providers prefer to equip their tools with a proprietary set of building elements generally referred to as Icons. Nevertheless, similar design principles are used since IN services, in this case, consist of appropriately initialised Icons chained together. 
A typical SCSE consists of the following components:

Designer: This module is equipped with a powerful graphical environment that allows the developer to connect a series of Icons in order to form a Service.

Version Manager: This module is equipped with a software engineering tool that allows the developer to keep track of the various service versions.

Compiler: This module is responsible for validating the existing service design and, if no errors are found, translate it into a high level language (usually $\mathrm{C} / \mathrm{C}++$ ).

Simulator: This module acts as a service switching point simulator based on the compiler produced code and allows the developer to model user behaviour by interacting with the service via a graphical interface. All relevant messages and errors are logged for debugging purposes.

A service is created by constructing a service picture on the Designer. Each such service picture consists of Icons joined by links. Each Icon encompasses a unique piece of functionality within the service. The starting point of each service is an Init Icon. On the other hand the service may terminate due to a variety of conditions therefore multiple terminating points are allowed denoted by respective Terminate Icons. Icons may produce results which denote the outcome of the operation performed by that Icon. These values can be stored in service variables. The user usually identifies such variables by a meaningful name. Apart from user defined variables, system pre-defined variables also exist. These pre-defined variables contain service related values i.e. the service trigger. The user may adapt an Icon's functionality to a specific service's needs by modifying the values of a set of Icon attributes known as Properties. The execution of the Service Logic always starts from the Init Icon and then follows the link to a next Icon depending on the result produced by the currently executed Icon. Icons performing a specific function can be grouped together in a special type of Icon called the Composite Icon. Composite Icons may contain other Composite Icons thus providing additional levels of navigation within the service picture. The service picture is completed with the definition of a set of Service Data Tables. These tables contain the run-time data which control the functionality of some of the Icons.

The transition from the formal description of the service to the creation environment requires a mapping procedure from SDL structures to Icons. This is necessary because the SDL specification includes characteristics of the DFP which in a high level design are transparent to the developer. A more detailed analysis of the problem can be found in (Bosco, 1994). Some general considerations regarding this transition are the following: (a) the identification of service functions that can be expressed as composite icons or as independent SLPs. The use of composite Icons makes the SLP more readable and therefore simpler to maintain, (b) the number of database transactions and logical comparisons to be performed during a service session. Forcing the service logic to perform a large number of unnecessary time consuming functions can severely affect performance and (c) the handling of exception conditions. Provision should be made for the handling of exception conditions that have been identified in the service requirements as well as usual 
programming exception conditions such as time-outs or failure to perform certain database transactions.

A proposed mapping of this type, aimed at the accommodation of the $\mathrm{VoD}$ and BVC service, is presented in the following list:

Init : This Icon initiates a service logic program. It defines whether the service is triggered by the calling or the called party's directory number.

Announce_Collect : This Icon handles the pair of PlayAndCollect and CollectedUserlnfo messages. It asks the B-IP to play the requested message and returns the user's response or in case of an error propagates an exception condition.

Script: This Icon handles the pair of ScriptRun and ScriptEvent messages. It asks the B-IP to execute the requested script and returns the script's result.

BearerOp : This Icon handles messages related to parties and bearers joining an existing session such as JoinPartyAndBearerToSession, ReleaseBearer etc. It returns the status of the operation.

Algorithm : This Icon performs variable related operations such as assign, increase, decrease, add, append etc. on a single variable.

Database : This Icon performs database transactions such as retrieve, write etc. on a single SDT and returns the result.

Condition : This Icon performs logical comparisons between values, variables etc. If the selected condition triggers then the Service Logic Program follows the leg occupied by the Condition Icon

Terminate : This Icon issues a ReleaseSession message and terminates the SLP.

\subsection{Icon based description of the VOD scrvice - scripts approach}

An image of the Service Provider Selection stage of the VOD service is presented in Figure 13. Each time user to service interaction must be performed the B-SCP demands from the B-IP the execution of the relevant script which contains the service logic code for the requested operation. This is done by sending a ScriptRun message from the B-SCP to the B-IP. The B-SCP then waits until an answer from the B-IP arrives, via a ScriptEvent, which should contain either the user's input or an error code.

The service image consists of just 7 Icons plus the relevant data tables. It is evident that the processing load on the B-SCP is significantly reduced compared to the case where we would be forced to include the entire SLP in the B-SCP. Another important factor which affects the performance an IN service is the number of database transactions which are performed during a service session. In this case a number of these operations has also been moved to the B-IP thus reducing even more the demand for processing power from the B-SCP.

1. Init : This Icon receives the ServiceRequest message. It defines that the service is triggered by the calling directory number.

2. Script : This Icon is used by the B-SCP in order to connect the user to the BIP, then instruct the B-IP to execute the Service Providers Selection script and finally to receive the script result. 
3. Condition : The occurrence of a failure to connect the user to the B-IP with respect to the previous operation is checked by this Icon. If such a situation arises then the SLP follows this leg.

4. Terminate : The session is terminated because of the failure that has occurred.

5. Condition : This Icon compares the script's result against the 'Success' code. If the selection has been carried out successfully then the SLP follows this leg and moves on to the next service stage.

6. BearerOp : The B-SCP instructs the B-SSP to disconnect the user from the B-IP.

7. Terminate : The session is terminated because the script did not return a 'Success' code.

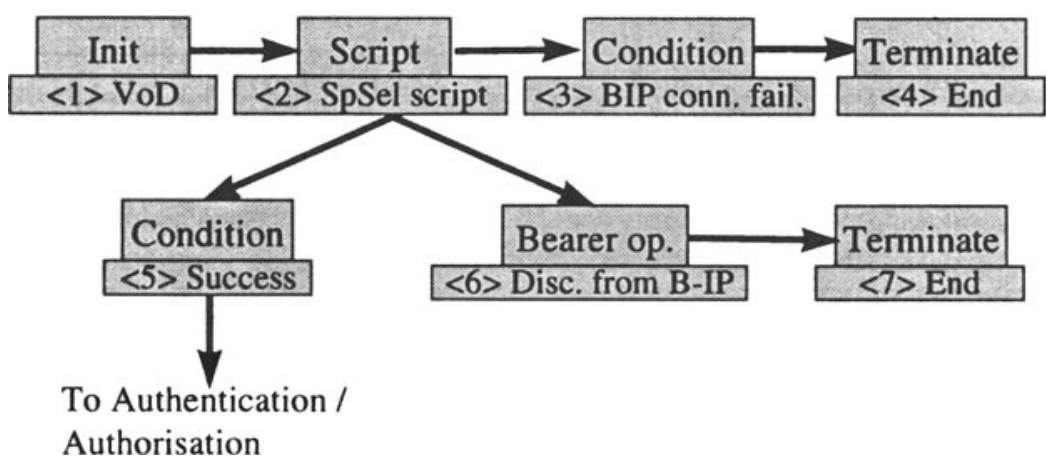

Figure 13 VoD Service Provider Selection description with Icons (scripts approach).

\subsection{Icon based description of the B-VC service}

An image of the B-VC service is presented in Figure 14. More specifically the Conference Creation procedure is examined.

1. Init : This is the starting point of the Conference Creation process

2. Database : The B-SCP searches the local service database in order to determine if the calling user is authorised.

3. Condition : The status of the database operation is checked for 'user record not found'. If such a status is returned then the SLP follows this leg.

4. Terminate : The session is terminated due to the failure of the database operation.

5. Announce_Collect : The B-SCP attempts to set-up a temporary User / B-IP connection then issues a PromptAndCollect message to the B-IP containing a create and lock conference command.

6. Condition : The status of the attempted connection is checked. If a failure has occurred then the SLP follows this leg. 
7. Terminate : The session is terminated because of the failure that occurred.

8. Database : The new conference record is created in the copy of the conference database that resides in the B-SCP.

9. Announce_Collect : The B-SCP issues a PromptAndCollect message to the B-IP containing an unlock conference command.

10. Condition : The status of the previous operation is checked for any exception condition. If an exception has indeed occurred then the SLP follows this leg.

11. BearerOp : The B-SCP instructs the B-SSP to disconnect the user from the B-IP.

12. Terminate : The Create Conference session is terminated.

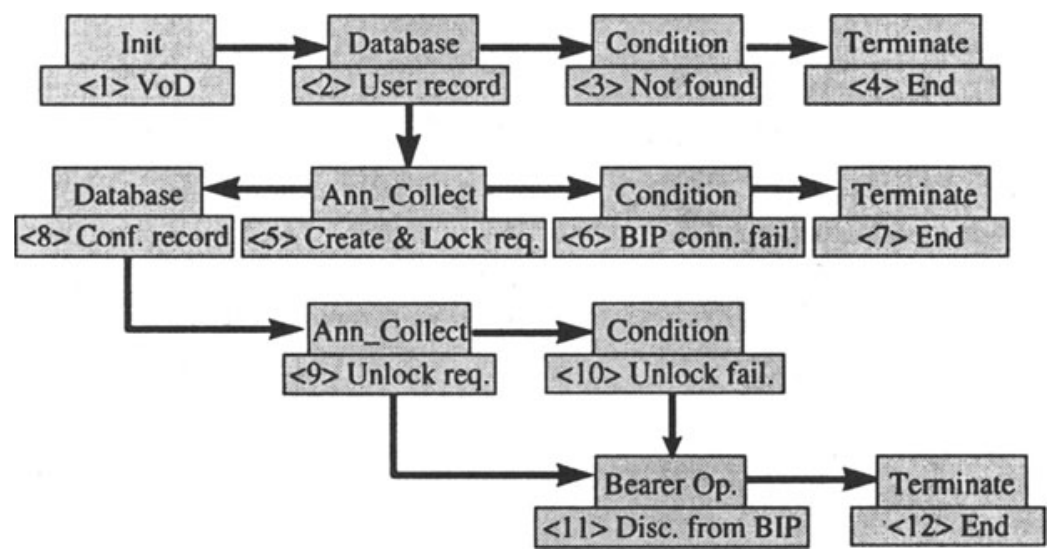

Figure 14 B-VC Conference Creation description with Icons.

\section{CONCLUSIONS}

In this paper we have proposed a complete methodology for the specification and design of typical IN based interactive multimedia services. This methodology is based on the GFP and DFP of the INCM and guarantees the disassociation of service provision from the network signalling capabilities. All stages of the service development process have been addressed and particular emphasis has been put to selected parts of the Service Logic for two IN services presented in detail by means of SDL diagrams and SCSE Icons. The proposed methodology has also been compared to other existing approaches in the field. Overall the service design approach is shown to be flexible enough to accommodate the requirements of a wide set of interactive multimedia services ranging from novel IN services to existing services migrating from other environments. 
The authors are currently engaged in the design and implementation of broadband IN-based services, that will be deployed in a European field trial demonstration over an ATM-based infrastructure.

\section{ACKNOWLEDGMENT}

This work has been partially funded by the ACTS project INSIGNIA (IN and BISDN Signalling Integration on ATM platforms). The opinions appearing are those of the authors and not necessarily of the other members of the consortium.

\section{REFERENCES}

Bosco G. P., F. Faraci F., (1994) Advanced intelligent network service laboratory, Canadian Journal of Electrical and Computer Engineering, January 1994.

Brandt H., Tittel C., Todorova P. (1997) Broadband Video Conference in an Integrated IN / B-ISDN Architecture, to be presented in NOC'97, Antwerp, Belgium, June 17-19 1997.

Doza Z. M., Lakhani H. A., Schraffnit T. L. (1990) A modular approach to intelligent networks: Telecom Canada's perspective, Proceedings of the 10th International Conference on Computer Communication, New Delhi India, 4-9 Nov. 1990.

GPT LIMITED UK (1996) GAIN 300 Inventor User Guide for release 304.2.

ITU-T Recommendation. I.210. Principles of Telecommunications services supported by an ISDN and the means to describe them.

ITU-T Recommendation Q.1201. Principles of Intelligent Network Architecture.

Thörner J. (1994) Intelligent Networks. Artech House, Boston, London.

ITU-T Recommendation Q.1213. Global Functional Plane for Intelligent Network CS-1.

ITU-T Recommendation Q1228.

ITU-T Recommendation Z.100. SLD Methodology Guidelines, Appendices I and II.

Kelly B., Crowther M., King J., Mason R., DeLapeyre J. (1995) Service validation and testing, Proceedings of Third International Workshop on Feature interactions in Telecommunication Software Systems, Kyoto Japan, 11-13 Oct. 1995.

Niitsu Y., Mizuno O., Okamoto M. (1992) Computer-aided stepwise service creation environment for intelligent network, Proceedings of SUPERCOMM/ACC'92, Chicago USA, 14-18 June 1992.

Nyeng A., Møller-Pedersen B. (1993) Approaches to the specification of Intelligent Network Services in SDL-92, in SDL'93 : Using Objects, Elsevier Science Publishers B. V. 
Olsen A., Nørbœk B (1995) Using SDL for Targeting Services to CORBA, Proceedings IS\&N '95, Heraclion, Greece, 16-20 October 1995.

Vezzoli L., Lorenzini L. (1996) Intelligent Mobile Video on Demand, Proceedings

ICIN '96, Bordeaux , France, 25-28 November 1996.

\section{BIOGRAPHY}

George N. Prezerakos was born in Athens, Greece. He received the Dipl. - Ing. Degree from the National Technical University of Athens (NTUA), Athens, Greece in 1993 in electrical and computer engineering. Since 1993 he is a Ph.D. candidate in NTUA and research associate of the Telecommunications Laboratory performing research in the area of Intelligent Networks, Service Engineering and Fuzzy Logic systems. His research interests are in the fields of service design and control for broadband networks. He has several publications in the above areas. He has participated in several European Union ACTS projects and is currently involved in the INSIGNIA AC068 project. Mr. Prezerakos is a member of the Technical Chamber of Greece.

Iakovos S. Venieris was born in Naxos, Greece. He received the Dipl. - Ing. degree from the University of Patras, Greece in 1988, and the Ph.D. degree from the National Technical University of Athens (NTUA), Athens, Greece, in 1990, all in electrical and computer engineering. From 1994 he is an Assistant Professor in the Electrical and Computer Engineering Department of NTUA. His research interests are in the fields of B-ISDN, signalling, resource scheduling and allocation for network management, modelling, performance evaluation and queueing theory. $\mathrm{He}$ has over sixty publications in the above areas. Dr. Venieris has been exposed to standardisation body work and has participated in several European Union and national projects dealing with B-ISDN protocols, Intelligent Networks, ATM switching and access techniques. Dr. Venieris is a member of IEEE and the Technical Chamber of Greece. 